\title{
Lern- und Leistungsziel- orientierung beim Übergang in die Sekundarstufe I: Längsschnittliche Befunde zur Bedeutung von Belastungen und Erziehungsverhalten von Eltern
}

\section{Markus P. Neuenschwander ${ }^{1}$}

Belastungen von Eltern beeinflussen elterliche Förderstrategien und die Lern- und Leistungszielorientierung von Kindern. Diese Hypothesen wurden mit Daten der Längsschnittstudie "Wirkungen der Selektion» (WiSel) überprüft. Es wurden 1096 Eltern und ihre Kinder im 6. und 7. Schuljahr der Sekundarstufe I (Gruppe 1) und 778 Eltern und ihre Kinder aus Kantonen mit Übertritt nach dem 6. Schuljahr (Gruppe 2, Vergleichsgruppe) befragt. Strukturgleichungsmodelle im Gruppenvergleich belegten, dass in beiden Gruppen gleicherweise belastete Eltern eher leistungsorientierten Druck ausübten. Leistungsorientierter Druck sagte die Leistungszielorientierung der Schülerinnen und Schüler vorher. Die Ergebnisse geben Hinweise, wie Eltern einer sinkenden Lern- und Leistungszielorientierung entgegenwirken.

\section{Einleitung}

Der Beitrag des elterlichen Verhaltens für den Schulerfolg von Kindern wurde immer wieder untersucht (z.B. Neuenschwander et al., 2005; Metaanalyse von Castro et al., 2015): Hohe Erwartungen, Kommunikation über Schulfragen und Förderung von Leseverhalten durch Eltern begünstigen die Entwicklung von Schülerleistungen. Zudem beeinflussen Eltern aufgrund ihres Erziehungsstils die Entwicklung der intrinsischen und extrinsischen Motivation der Kinder in der Schule (Grolnick, 2009; Neuenschwander \& Frank, 2011). Diese Zusammenhänge wurden unter der Bedingung hoher Belastungen von Eltern und unter der Bedingung hoher Belastungen von Kindern, die bei einem Schulübergang auftreten können, seltener untersucht. Wie sehr beeinflussen Belastungen von Eltern ihr Erziehungsverhalten und die Entwicklung kognitiver Motivationsaspekte wie der Lernzielorientierung und der Leistungszielorientierung ihrer Kinder in der Sekundarstufe I und beim Übergang in die Sekundarstufe I? 


\section{Erziehungsverhalten und Lern- und Leistungsziel- orientierung}

Die Forschung zur Leistungsorientierung als Strang der kognitiven Motivationsforschung erhielt in den letzten Jahren hohe Aufmerksamkeit. Pintrich, Conley und Kempler (2003) beschrieben die Leistungsorientierung (achievement orientation) als kognitive Repräsentation davon, was Individuen zu lernen oder zu leisten versuchen. Leistungsorientierung wird oft differenziert in Lernzielorientierung (mastery goals) und Leistungszielorientierung (performance goals). Lernzielorientierung fokussiert auf den Erwerb von Kompetenzen und das Verstehen von Aufgaben, was mit intrinsischer Motivation verbunden ist (Grolnick, 2009). Leistungszielorientierung bezieht sich hingegen auf das Zeigen von Kompetenzen, die Verwendung von sozial vergleichenden und normativen Standards (vgl. Neuenschwander, im Druck), was mit extrinsischer Motivation einhergeht (Grolnick, 2009). Während die Korrelationen zwischen Lernziel- bzw. Leistungszielorientierung und Leistungstestergebnissen bzw. Noten in der Regel klein sind oder fehlen, erklären Kombinationen von Lern- und Leistungszielorientierung im Jugendalter Leistungstestergebnisse und Noten bedeutsam (Conley, 2012).

Die Forschung zu parental involvement belegte immer wieder die zentrale Bedeutung der Eltern für die Entwicklung der Lern- und Leistungszielorientierung ihrer Kinder (Grolnick, Friendely \& Bellas, 2009). Der Zusammenhang zwischen dem Erziehungsverhalten von Eltern und der Lern- und Leistungszielorientierung ist aber bisher selten untersucht worden (Gonzalez-DeHass, Willems \& Holbein, 2005). Das Erziehungsverhalten von Eltern wurde in vielerlei Hinsicht systematisiert. Es werden zwei Erziehungsstile unterschieden (Wild, 1999): Ein autonomieorientierter Erziehungsstil begünstigt das Ziel von Kindern, sich autonom mit einem Lerninhalt auseinanderzusetzen. Damit wird den Kindern wichtig, einen Inhalt genau zu verstehen. Ein leistungsorientierter Erziehungsstil begünstigt hingegen das Ziel von Kindern, die eigene Leistung zeigen zu wollen, sich mit den eigenen guten Leistungen zu präsentieren. Wenn Eltern Leistungsdruck ausüben, wird es Kindern wichtig zu zeigen, dass sie die Leistungsanforderungen der Eltern erfüllen (Gurland \& Grolnick, 2005; Régner, Loose \& Dumas, 2009). Daraus werden folgende Hypothesen abgeleitet:

1. Leistungsorientierter Druck von Eltern während der Sekundarstufe I begünstigt (1a) die Leistungszielorientierung von Kindern, aber (1b) nicht die Lernzielorientierung.

2. Autonomieorientierte Hilfe von Eltern während der Sekundarstufe I begünstigt nicht (2a) die Leistungszielorientierung, aber (2b) die Lernzielorientierung von Kindern.

\section{übergang in die Sekundarstufe I}

Der Schulübergang in die Sekundarstufe I beginnt mit einer Beurteilungsphase, die in einem Entscheid für ein Schulniveau resultiert. Danach müssen sich Jugendliche beim Schulwechsel an eine neue Schulorganisation und Klassen- 
zusammensetzung anpassen. Diese Schritte sind mit Unsicherheiten und Belastungen von Kindern verbunden (Sirsch, 2000). Wir wissen wenig, ob sich der Erziehungsstil der Eltern auf die Lern- und Leistungszielorientierung bei einem Schulübergang gleich wie in der Regelschulsituation (d.h. nicht während eines Schulübergangs) auswirkt. Duchesne und Ratelle (2010) zeigten, dass elterliche Kontrolle vermittelt über die Schülerangst die Leistungszielorientierung nach dem Schulübertritt verstärkt. Die Theorie der Erziehungsstile geht von der Universalitätsannahme aus, wonach Erziehungsstile unabhängig von der Schulsituation die Lern- und Leistungszielorientierung in der Kindheit beeinflussen (Wild, 1999). Es wird daher angenommen, dass analog zur Situation in der Sekundarstufe I auch während des Schulübergangs der leistungsorientierte Druck von Eltern die Leistungszielorientierung bzw. die autonomieorientierte Hilfe der Eltern die Lernzielorientierung beeinflusst. Es werden konkret folgende Hypothesen überprüft:

3. In Schulübergängen beeinflusst der leistungsorientierte Druck von Eltern (3a) die Leistungszielorientierung, (3b) aber nicht die Lernzielorientierung.

4. In Schulübergängen beeinflusst der autonomieorientierte Hilfe von Eltern (4a) nicht die Leistungszielorientierung, (4b) aber die Lernzielorientierung.

5. Die Zusammenhänge von Erziehungsstil und Lern- und Leistungszielorientierung sind in der Regelschulsituation der Sekundarstufe I gleich wie in der Situation des Schulübergangs (Stabilität der Zusammenhänge in verschiedenen Situationen).

\section{Elternbelastungen und Erziehungsverhalten}

Frühere Studien zeigten, dass Belastungen das Erziehungsverhalten von Eltern beeinträchtigen. Belastungen von Eltern treten wegen beruflichen Konflikten, Problemen in der Familie, Konflikten mit ihrem Kind oder persönlichen, ungelösten Problemen auf (Newland, Crnic, Cox, Mills-Koonce \& Family Life Project Key, 2013) und senken die Sensibilität der Eltern gegenüber den Bedürfnissen ihrer Kinder. Bereits Roberts (1989) zeigte, dass ausgeprägte Belastungen von Eltern negativ mit kindbezogenen Elternmerkmalen wie Wärme und Responsivität zusammenhängen.

Aufgrund dieser Befunde wird vermutet, dass belastete Eltern ihren Kindern bei schulischen Aufgaben weniger empathisch und flexibel Hilfestellungen geben und mehr Mühe haben, für sie einen unterstützenden Raum für eigene Problemlösungen zu schaffen. Hohe Belastungen von Eltern begünstigen eher kontrollierendes und strukturierendes Erziehungsverhalten (Gurland \& Grolnick, 2005). Eltern in Belastungssituationen erziehen eher mit starren Regeln und verschaffen ihren Kindern ein klar organisiertes, eher kühles Umfeld, in dem sich die Eltern mit wenig emotionalem Engagement bewegen können. Daraus wird die sechste Hypothese abgeleitet:

6. Belastete Eltern vermitteln (6a) ihren Kindern mehr leistungsorientierten Druck und gewähren $(6 \mathrm{~b})$ weniger autonomieorientierte Hilfe. 


\section{Methode}

Die formulierten Hypothesen wurden im Rahmen des Projekts Wirkungen der Selektion (WiSel) überprüft. Sowohl die Schülerinnen und Schüler als auch deren Eltern wurden mit einem standardisierten Fragebogen befragt.

\section{Stichprobe}

\section{Schülerbefragung}

Im Jahr 2011 wurden insgesamt 99 Schulklassen mit Kindern des 5. Schuljahres aus vier Kantonen zufällig ausgewählt (Tabelle 1). Diese Kinder und ihre Eltern wurden im 6. und 7. Schuljahr erneut befragt. Die vorliegende Auswertung basiert auf den Fragebogendaten der Kinder und ihrer Eltern im 6. und 7. Schuljahr. Es nahmen 541 Schülerinnen und 555 Schüler aus den Kantonen Aargau und Basel-Landschaft an der Studie teil, die sich im 6. Schuljahr bereits in der Sekundarstufe I befanden (Gruppe Sekundarstufe I). Zudem nahm eine Vergleichsgruppe mit 383 Schülerinnen und 395 Schülern aus den Kantonen Bern und Luzern mit der Transition in die Sekundarstufe I im Anschluss an das 6. Schuljahr teil (Gruppe Transition). Die Kinder waren im 6. Schuljahr durchschnittlich 12jährig und hatten in $82 \%$ der Fälle eine Schweizer Staatsangehörigkeit. Rund 23\% der Kinder besuchten ein Schulniveau mit Grundanforderungen, 40\% ein Schulniveau mit erweiterten Anforderungen, 36\% ein Schulniveau mit hohen Anforderungen (progymnasial).

Tabelle 1: Stichprobendesign und Stichprobengrössen

\begin{tabular}{llllll}
\hline & 5. Klasse & 6. Klasse & 7. Klasse & $\mathrm{N}_{\text {(weibl) }}$ & $\mathrm{N}_{\text {(männl) }}$ \\
\hline $\begin{array}{l}\text { Gruppe Sekundarstufe I } \\
\text { (Kt AG/BL) }\end{array}$ & Primar & Sek I & Sek I & 541 & 555 \\
$\begin{array}{l}\text { Gruppe Transition } \\
\text { (Kt BE/LU) }\end{array}$ & Primar & Primar & Sek I & 383 & 395 \\
\hline
\end{tabular}

Legende: Kt AG/BL: Kantone Aargau/Basel-Landschaft, Kt BE/LU: Kantone Bern/Luzern

Um systematische Rücklaufverzerrungen zu überprüfen, wurden die in dieser Analyse verwendeten Variablen zwischen den Schülern und Schülerinnen, die nur im 6. Schuljahr befragt worden sind, mit denen verglichen, die im 6. und 7. Schuljahr befragt worden sind. Es fanden sich nur sehr geringe systematische Rücklaufverzerrungen bei den Schüler- oder Elterndaten (Belastungen der Eltern: $F(1,1858)=7.9, p<.01, \mathrm{~d}=.15$; autonomieorientierte Hilfe der Eltern: $F(1,1885)=.04, \mathrm{~ns}, \mathrm{~d}=.01$; leistungsorientierter Druck der Eltern: $F(1,1884)$ $=5.1, p<.05, \mathrm{~d}=.10$; Leistungszielorientierung: $F(1,1976)=4.4, p<.05, \mathrm{~d}=$ .09 , Lernzielorientierung: $F(1,1979)=.53$, ns, $\mathrm{d}=.04)$. 


\section{Elternbefragung aus WiSel Stichprobe}

Die wichtigste Bezugsperson mit Erziehungsverantwortung der Jugendlichen im 6. Schuljahr füllte den Elternfragbogen aus (Mutter: 82\%, Vater: 17\%, Andere: 1\%; Durchschnittsalter: 43.1 Jahre; Anteil Schweizer Staatsbürgerschaft: 84\%). Von $95.5 \%$ der befragten Jugendlichen lagen ausgefüllte Elternfragebogen vor, was einem sehr hohen Rücklauf des Elternfragebogens entspricht.

\section{Instrument}

Schülerbefragung

Die Lernzielorientierung wurde mit drei Items von Midgley et al. (2000) gemessen, wie zum Beispiel «Mir ist es wichtig, die im Unterricht behandelten Themen vollständig zu verstehen». Es wurde eine Ratingskala mit 6 Punkten (1 'trifft überhaupt nicht zu' und 6 'trifft voll und ganz zu') verwendet $(M=5.162$, $S D=.67, \alpha=.62$ ).

Die Leistungszielorientierung wurde mit drei Items von Midgley et al. (2000) gemessen wie zum Beispiel «Es ist mir wichtig, im Vergleich mit den Anderen in meiner Klasse klug zu wirken». Es wurde eine Ratingskala mit 6 Punkten (1 'trifft überhaupt nicht zu' bis 6 'trifft voll und ganz zu') verwendet $(M=3.62$, $S D=1.19, \alpha=.80)$.

\section{Elternbefragung}

Die Skala wahrgenommener Stress von Cohen, Kamarck und Mermelstein (1983) setzte sich aus 5 Items zusammen, wie zum Beispiel «Wie häufig hatten Sie im letzten Monat das Gefühl, dass sich Schwierigkeiten so anhäufen, dass Sie sie nicht mehr bewältigen können?» $(M=2.19, S D=.55, \alpha=.67)$. Die 6-Punkte Rating Skala hatte die Pole 1 'nie' und 6 'fast immer'.

Die Skala Erziehungsverhalten von Eltern von Wild, Remy, Gerber und Exeler (2001) wurde faktorenanalytisch in zwei Faktoren gegliedert und auf einer 6-Punkte Ratingskala mit den Polen 1 'stimmt überhaupt nicht' und 6 'stimmt voll und ganz' beantwortet. Der Faktor Autonomieunterstützende Hilfe $(M=5.08, S D=.58, \alpha=.68)$ umfasste drei Items wie zum Beispiel «Wenn ich meinem Kind bei den Hausaufgaben helfe, ermuntere ich es, erst einmal selbst die richtige Lösung zu finden». Der Faktor Leistungsorientierter Druck $(M=2.95$, $S D=1.02, \alpha=.77)$ umfasste drei Items wie zum Beispiel «Bei einer schlechten Note/Leistungsbeurteilung schimpfe ich mit meinem Kind und verlange von ihm, mehr zu tun.»

\section{Durchführung}

Vor der Datenerhebung wurde das schriftliche Einverständnis der kantonalen Verwaltung, der Schulleitung, der Lehrpersonen und der Eltern zur Teilnahme an der Studie eingeholt. Die Datenerhebung fand zu beiden Messzeitpunkten zwischen Oktober und Dezember statt, d.h. die Befragung in der Gruppe Transition fand statt, bevor der Selektionsentscheid gefällt worden ist. Die 
Datenerhebung in den Schulklassen wurde jeweils von geschulten Mitarbeiterinnen und Mitarbeitern des Projektteams während des Regelunterrichts durchgeführt. Die Schülerinnen und Schüler füllten während 90 Minuten einen Fragebogen aus und nahmen den Elternfragebogen mit nach Hause. Der Elternfragebogen wurde von einer Projektmitarbeiterin nach ca. 3 Wochen bei einem zweiten Klassenbesuch eingesammelt.

\section{Auswertungsverfahren}

Im ersten Schritt wurden getrennt für die Kinder, die sich bereits in der Sekundarstufe I befanden (Gruppe Sekundarstufe I) und für die Kinder, die sich im Übertrittsverfahren befanden (Gruppe Transition), die Interkorrelationen gerechnet.

Die Hypothesen wurden mit Strukturgleichungsmodellen mit der Software Mplus 7.3 (Muthén \& Muthén, 1998/2012) überprüft. Die Full Information Maximum Likelihood (FIML) Prozedur in MPlus wurde angesichts von Missing values verwendet. Die Variablen im 6. Schuljahr wiesen einen Missinganteil von 2.9\%-6.5\% auf. Die Lern- und Leistungszielorientierung im 7. Schuljahr hatten einen Missinganteil von je 52\%. Die FIML Prozedur führt zu reliablen Schätzwerten (Graham, 2009). Um die Verschachtelung der Schülerinnen und Schülern in Schulklassen abzubilden, wurde die Klassenzugehörigkeit im 6. Schuljahr kontrolliert (type=complex). Dieses Verfahren führt zu analogen Ergebnissen wie Mehrebenenanalysen. Es wird der $\chi^{2}$-Wert erwähnt, obwohl er in der vorliegenden grossen Stichprobe dazu neigt, signifikant zu werden. Ein nicht signifikanter $\chi^{2}$-Wert verweist auf einen guten Fit zwischen dem Modell und den Daten. Der $\chi^{2}$-Wert erlaubt auch, die Anpassungsgüte zweier Modelle zu vergleichen. Wegen des verschachtelten Designs werden $\chi^{2}$-Werte berechnet, deren Differenzen nicht $\chi^{2}$ verteilt sind. Es wird daher der Santorra-Bentler skalierte $\chi^{2}$-Differenz-Test ausgewiesen.

\section{Ergebnisse}

\section{Deskriptive Ergebnisse}

Im ersten Schritt werden die Pearson-Korrelationen zwischen den involvierten Variablen, getrennt für die beiden Gruppen Transition und Sekundarstufe I, dargestellt (Tabelle 2). Die Korrelationen zwischen Leistungszielorientierung und Lernzielorientierung liegen im Bereich .19 $\leq r \leq .24$. Die Elternbelastungen korrelierten mit der Lernzielorientierung in der Gruppe Sekundarstufe I signifikant positiv, aber nicht mit der Leistungszielorientierung. In der Gruppe Transition korrelierten die Elternbelastungen mit der Lern- und Leistungszielorientierung hingegen nicht signifikant.

Danach wurden die Mittelwerte aller verwendeten Variablen im 6. Schuljahr zwischen den beiden Kohorten varianzanalytisch verglichen (Tabelle 3). Es fand 
sich nur bei der Variable leistungsorientierter Druck ein signifikanter Gruppenunterschied $(F(1,1775)=8.5, p<.01)$, wonach die Eltern von Kindern in der Gruppe Transition über mehr Ausübung von leistungsorientiertem Druck berichteten als diejenigen von gleichaltrigen Kindern in der Gruppe Sekundarstufe I.

Schliesslich wurden die Veränderungen der Lern- und Leistungszielorientierung vom 6. zum 7. Schuljahr zwischen der Gruppe Transition mit der Gruppe Sekundarstufe I verglichen. Varianzanalysen mit Messwiederholung zeigten für die Leistungszielorientierung einen signifikanten Zeiteffekt $(F(1$, $868)=30.7, p<.001)$, aber keinen Gruppen- oder Interaktionseffekt. Die Leistungszielorientierung nahm sowohl während des Übergangs als auch in der Sekundarstufe I signifikant ab. Im 7. Schuljahr war aber die Leistungszielorientierung in der Gruppe Sekundarstufe I höher als in der Gruppe Transition $(F(1$, $893)=4.3, p<.05)$. Für die Lernzielorientierung war der Zeiteffekt $(F(1,871)$ $=51.2, p<.0011)$ signifikant. Der Gruppeneffekt und die Interaktion waren nicht signifikant. Die Lernzielorientierung nahm ebenfalls in beiden Gruppen bedeutsam ab. Im 7. Schuljahr war aber die Lernzielorientierung in der Gruppe Sekundarstufe I höher als in der Gruppe Transition $(F(1,894)=4.1, p<.05)$.

Tabelle 2: Interkorrelationen

\begin{tabular}{|c|c|c|c|c|c|c|c|}
\hline & 1 & 2 & 3 & 4 & 5 & 6 & 7 \\
\hline $1 \mathrm{~S}$ Leistungsziel $\mathrm{t}_{1}$ & - & $.50^{* * *}$ & $.22^{* * *}$ & $.15^{* * *}$ & .03 & .10 & .01 \\
\hline $2 S$ Leistungsziel $t_{2}$ & $.52^{* * *}$ & - & .06 & $.19^{* * *}$ & .02 & $.24^{* * *}$ & .01 \\
\hline $3 S_{\text {Lernziel }}{ }_{1}$ & $.20^{* * *}$ & $.15^{* * *}$ & - & $.48^{* * *}$ & $.17^{* * *}$ & .07 & .02 \\
\hline $4 \mathrm{~S}$ Lernziel $t_{2}$ & $.12^{* *}$ & $.24^{* * *}$ & $.55^{* * *}$ & - & $.15^{* *}$ & $.15^{* *}$ & -.01 \\
\hline 5 E Elternbelastung $\mathrm{t}_{1}$ & -.02 & .05 & .03 & $.11^{*}$ & - & -.05 & $-.21^{* * *}$ \\
\hline 6 E leistungsorientierter Druck $t_{1}$ & $.17^{* * *}$ & $.16^{* * *}$ & -.01 & -.01 & -.09 & - & $.24^{* * *}$ \\
\hline $7 \mathrm{E}$ autonomieorientierte Hilfe $\mathrm{t}_{1}$ & .09 & $.11^{*}$ & .02 & .02 & $-.20^{* * *}$ & $.26^{* * *}$ & - \\
\hline
\end{tabular}

Legende: unter der Diagonale: Gruppe Transition, über der Diagonale: Gruppe Sekundarstufe I, S: Schülerdaten, E: Elterndaten, ; $\mathrm{t}_{1}: 6$. Schuljahr, $\mathrm{t}_{2}: 7$. Schuljahr

${ }^{*}: p<.05,{ }^{* *}: p<.01,{ }^{* * *}: p<.001$

\section{Tabelle 3: Beschreibung der Mittelwerte differenziert nach Gruppe}

\begin{tabular}{lccl}
\hline & $\begin{array}{c}\text { Gruppe } \\
\text { Transition }\end{array}$ & $\begin{array}{c}\text { Gruppe } \\
\text { Sekundarstufe } \mathrm{I}\end{array}$ & $F, \mathrm{df}_{1}, \mathrm{df}_{2}, p$ \\
\hline Elternbelastungen $\mathrm{t}_{1}$ & 2.18 & 2.19 & $0.1,1,1751, n s$ \\
E: leistungsorientierter Druck $\mathrm{t}_{1}$ & 2.85 & 2.99 & $8.5,1,1775, p<.01$ \\
E: autonomieorientierte Hilfe $\mathrm{t}_{1}$ & 5.08 & 5.06 & $.2,1,1751$ ns \\
Leistungsziel $\mathrm{t}_{1}$ & 3.40 & 3.47 & $1.5,1,1815, n s$ \\
Leistungsziel $\mathrm{t}_{2}$ & 3.07 & 3.24 & $4.3,1,893, p<.05$ \\
Lernziel $\mathrm{t}_{1}$ & 5.09 & 5.04 & $2.2,1,1817, n s$ \\
Lernziel $_{2}$ & 4.96 & 4.86 & $4.1,1,894, p<.05$ \\
\hline
\end{tabular}

Legende: $t_{1}: 6$. Schuljahr, $t_{2}: 7$. Schuljahr 


\section{Invarianzanalysen}

Um die Invarianz der verwendeten Konzepte zwischen den beiden Gruppen zu testen, wurden nach dem Verfahren von van de Schoot, Lugtig und Hox (2012) Invarianzanalysen unter Berücksichtigung der Verschachtelung der Daten in Schulklassen durchgeführt. Zuerst wurden für die Lernziel- und die Leistungszielorientierung für beide Gruppen die Invarianzanalysen im Vergleich der beiden Messzeitpunkte und danach im Gruppenvergleich durchgeführt (vgl. Little, 2013). Im nächsten Schritt wurde die Invarianz der Konzepte zu den Eltern zwischen den Gruppen getestet. Die Invarianzanalysen wurden jeweils in drei Schritten durchgeführt. (1) Zur Berechnung der konfiguralen Invarianz wurden die Ladungen und Intercepts der Indikatoren zwischen den Gruppen frei geschätzt. Die Mittelwerte der Faktoren wurden aber auf null fixiert. (2) Die metrische (schwache) Invarianz wurde getestet, indem die Faktorladungen der jeweils gleichen Items zwischen den Gruppen bzw. Messzeitpunkten gleichgesetzt wurden. (3) Zusätzlich wurden dann die Intercepts der Indikatoren zwischen den Gruppen bzw. Messpunkten gleich gesetzt, um die skalare (starke) Invarianz zu testen.

Zuerst wurde das Modell mit konfiguraler Invarianz für die Lernziel- und Leistungszielorientierung im 5. und 6. Schuljahr für beide Gruppen getestet. Die Ladungen zwischen den Gruppen wurden frei geschätzt. Die latenten Variablen korrelierten miteinander. Die jeweils gleichen Items korrelierten zwischen den Messzeitpunkten. Das Modell erreichte einen guten Fit $\left(\chi^{2}=189.7, \mathrm{df}=104\right.$, $p<.001, \mathrm{CFI}=.98, \mathrm{RMSEA}=.03)$. Seine Anpassungsgüte verschlechterte sich gegenüber dem Modell mit metrischer Invarianz $\left(\chi^{2}\right.$ change $\left.=5.76, \mathrm{df}=4, n s\right)$ nicht signifikant. Die Anpassungsgüte dieses Modells verschlechterte sich aber gegenüber dem Modell mit skalarer Invarianz signifikant $\left(\chi^{2}\right.$ change $=27.1, \mathrm{df}$ $=2, p<.001)$. Die Konzepte erfüllten über die beiden Messzeitpunkte die Anforderungen der metrischen Invarianz.

Danach wurde das Modell mit konfiguraler Invarianz zwischen den Gruppen für die Lern- und Leistungszielorientierung gerechnet $\left(\chi^{2}=183.3\right.$, df $=96$, $\mathrm{p}<.001, \mathrm{CFI}=.98, \mathrm{RMSEA}=.03)$. Die Anpassungsgüte verschlechterte sich im Vergleich zum Modell mit metrischer Invarianz nicht signifikant $\left(\chi^{2}\right.$ change $=6.7, \mathrm{df}=8, n s)$, aber zum Modell mit skalarer Invarianz $\left(\chi^{2}\right.$ change $=31.1$, $\mathrm{df}=12, p<.001)$. Wenn die Intercepts von je einem Item pro Konzept und Messzeitpunkt (d.h. vier Items) zwischen den Gruppen fixiert wurden, nahm der Fit nicht signifikant $\mathrm{ab}\left(\chi^{2}\right.$ change $\left.=6.11, \mathrm{df}=4, n s.\right)$. Es liegt zwischen den Gruppen eine partielle skalare Invarianz vor.

Im nächsten Schritt wurde die Invarianz der Elternkonzepte zwischen den Gruppen verglichen. Die latenten Variablen korrelierten frei. Das Modell mit konfiguraler Invarianz erreichte einen guten Fit $\left(\chi^{2}=190.3 \mathrm{df}=100, p<.001\right.$, CFI $=.97$, RMSEA $=.03)$. Der Fit verschlechterte sich im Vergleich zum Modell mit metrischer Invarianz nicht signifikant $\left(\chi^{2}\right.$ change $\left.=5.4, \mathrm{df}=9, n s\right)$. Dieser Fit verschlechterte sich im Vergleich zum Modell mit skalarer Invarianz 
auch nicht signifikant $\left(\chi^{2}\right.$ change $\left.=20.4, \mathrm{df}=12, n s\right)$. Die Konzepte haben also zwischen den Gruppen eine skalare Invarianz.

Die Invarianzanalysen zeigen, dass zumindest metrische, z.T. partielle oder vollständige skalare Invarianz vorliegt. Damit sind die Voraussetzungen für das Berechnen von Strukturgleichungsmodellen, in welchen Zusammenhänge zwischen latenten Variablen geschätzt werden, erfüllt.

\section{Model lprüfung}

Im nächsten Schritt wurden für die Gruppen Transition und Sekundarstufe I die postulierten Strukturgleichungsmodelle für die Leistungszielorientierung definiert und miteinander verglichen. In beiden Gruppen wurden die wahrgenommene Elternbelastung gemäss der Hypothesen 6a und 6b zur Vorhersage der autonomieorientierten Hilfe und des Leistungsdrucks verwendet. Zur Prüfung der Hypothesen 1a und 2a wurden von diesen latenten Variablen Pfade auf die Leistungszielorientierung im 7. Schuljahr zugelassen, die ihrerseits durch die Leistungszielorientierung im 6 . Schuljahr vorhergesagt wurde. Es wurde kein direkter Pfad von den Elternbelastungen auf die Leistungszielorientierung geschätzt, weil die Korrelation gemäss Tabelle 1 nicht signifikant war. Die Ladungen der Items wurden zwischen den Gruppen gleich gesetzt. Dieses Modell ohne Restriktionen zwischen den latenten Variablen der beiden Gruppen erreichte eine hohe Anpassungsgüte $\left(\chi^{2}=429.4, \mathrm{df}=278, p<.001\right.$, CFI $=.98$, RMSEA $=.02)$. Die Fit-Indices des Vergleichsmodells, in dem die Koeffizienten zwischen den latenten Variablen zwischen den Gruppen fixiert waren, waren nicht schlechter $\left(\chi^{2}=431.3, \mathrm{df}=283, p<.001\right.$, CFI $=.98$, RMSEA $=.02, \chi^{2}$ change $=2.61(5)$, ns). Die Parameter unterschieden sich daher gemäss Hypothese 5 nicht zwischen den Gruppen. Gemäss Abbildung 1 erklärten die Elternbelastungen, in Übereinstimmung mit den Hypothesen 6a und b, die autonomieorientierte Hilfe negativ und den Leistungsdruck positiv. Der Leistungsdruck erklärte gemäss Hypothese 1a die Leistungszielorientierung im Folgejahr nach Kontrolle der Leistungszielorientierung im vorangehenden Jahr signifikant. Die autonomieorientierte Hilfe erklärte die Leistungszielorientierung nicht signifikant, aber die Lernzielorientierung (Hypothese 2a, 2b). In Übereinstimmung mit Hypothesen 3a und 4a sowie Hypothese 5 unterschied sich das Muster der Pfade zwischen der Gruppe Transition und der Gruppe Sekundarstufe I nicht. 

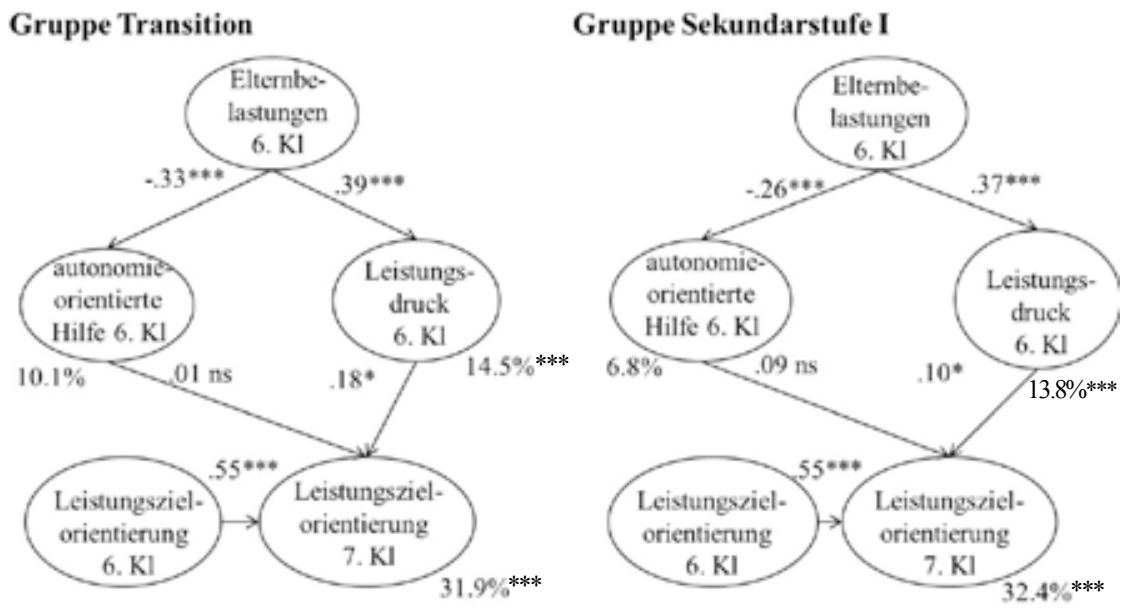

Abbildung 1: Strukturgleichungsmodell zu Leistungszielorientierung im Gruppenvergleich mit Angabe der Varianzaufklärung neben den latenten abhängigen Variablen

$\left(^{*}: p<.05,{ }^{* *}: p<.01,{ }^{* * *}: p<.001, \chi^{2}=429.4, \mathrm{df}=278, p<.001, \mathrm{CFI}=.98, \mathrm{RMSEA}=.02\right.$,

$\mathrm{N}_{\text {Gruppe Transition }}=777, \mathrm{~N}_{\text {Gruppe Sekundarstufe I }}=1089$ )

Im nächsten Schritt wurden für die Gruppen Transition und Sekundarstufe I die postulierten Strukturgleichungsmodelle für die Lernzielorientierung definiert und miteinander verglichen. Es wurde kein direkter Pfad von den Elternbelastungen auf die Lernzielorientierung geschätzt, weil die Korrelation gemäss Tabelle 1 nicht signifikant war. In beiden Gruppen wurden gemäss Hypothesen $6 \mathrm{a}$ und $6 \mathrm{~b}$ die wahrgenommenen Elternbelastungen zur Vorhersage der autonomieorientierten Hilfe und des Leistungsdrucks verwendet. Diese beiden latenten Variablen sagten gemäss Hypothesen $1 \mathrm{~b}$ und $2 \mathrm{~b}$ die Lernzielorientierung im 7. Schuljahr nach Kontrolle der Lernzielorientierung im 6. Schuljahr vorher. Die Ladungen der Messmodelle wurden zwischen den Gruppen fixiert. Das Modell ohne Restriktionen der Korrelationen zwischen den latenten Variablen und zwischen den Gruppen erreichte eine hohe Güte $\left(\chi^{2}=460.3, \mathrm{df}=278, p\right.$ $<.001$, CFI $=.97$, RMSEA = .03). Die Fit-Indices des Vergleichsmodells, in dem die Koeffizienten des Strukturmodells zwischen den Gruppen fixiert waren, erreichte ähnliche Gütekriterien $\left(\chi^{2}=465.0, \mathrm{df}=283, p<.001\right.$, CFI $=.97$, RMSEA $=.03, \chi^{2}$ change $\left.=4.64, \mathrm{df}=5, n s\right)$. Die Parameter des Strukturmodells unterschieden sich daher gemäss Hypothese 5 zwischen den Gruppen nicht signifikant. Gemäss Abbildung 2 erklärten die Elternbelastungen in Übereinstimmung mit den Hypothesen 6a und 6b die autonomieorientierte Hilfe negativ und den Leistungsdruck positiv. Die autonomieorientierte Hilfe erklärte gemäss Hypothese 2b die Lernzielorientierung in der 7. Klasse nach Kontrolle 
der Lernzielorientierung in der 6. Klasse positiv - wenn auch nur in der Gruppe Sekundarstufe I signifikant. Der leistungsorientierte Druck erklärt die Lernzielorientierung in der Gruppe Transition signifikant, aber nicht in der Gruppe Sekundarstufe I (abweichend zu Hypothese 3b). In dieser Situation war autonomieorientierte Hilfe für die Lernzielorientierung günstiger (Hypothese 4b). Obwohl sich die Signifikanzen aufgrund unterschiedlicher Standardmessfehlern zwischen den beiden Gruppen teilweise unterschieden, unterschieden sich die Parameter des Modells zwischen den Gruppen insgesamt nicht signifikant.
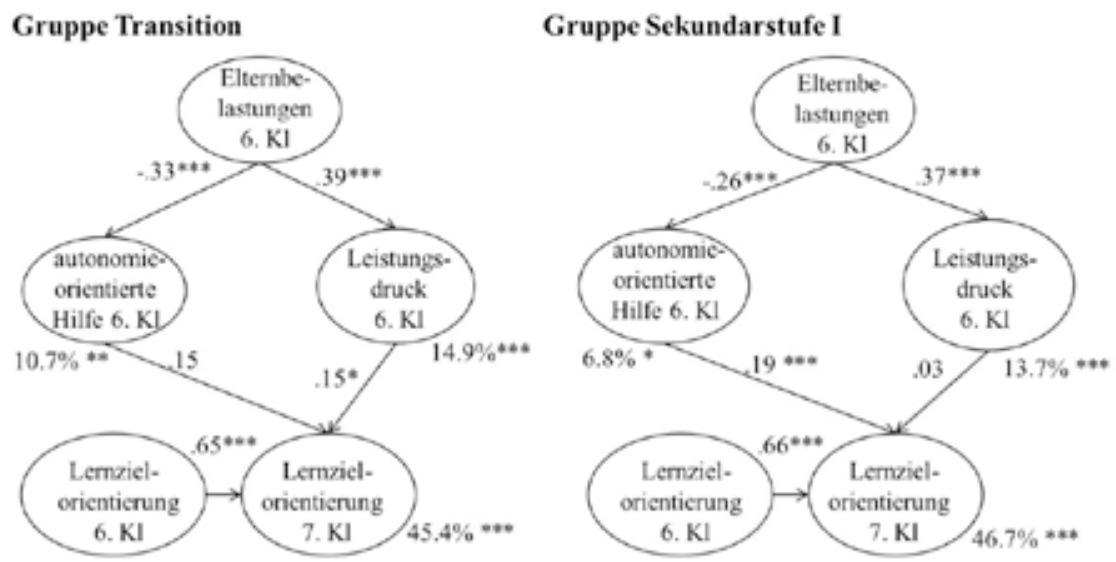

Abbildung 2: Strukturgleichungsmodell zu Lernzielorientierung im

Gruppenvergleich mit Angabe der Varianzaufklärung neben den latenten abhängigen Variablen

$\left(*: p<.05,{ }^{* *}: p<.01,{ }^{* *}: p<.001, \chi^{2}=460.3, \mathrm{df}=278, p<.001, \mathrm{CFI}=.97, \mathrm{RMSEA}=.03, \mathrm{~N}_{\text {Gruppe }}\right.$ Transition $=777, \mathrm{~N}_{\text {Gruppe Sekundarstufe I }}=1090$ )

\section{Diskussion}

Die längsschnittlichen Ergebnisse lassen sich in die Forschung zum Beitrag von Eltern zur Entwicklung der Lernziel- und Leistungszielorientierung vor und nach Eintritt in die Sekundarstufe I einordnen (Midgley, Kaplan \& Middelton, 2001). Frühere Forschung zeigte, dass die Lern- und Leistungsmotivation von Schülerinnen und Schülern wichtige Determinanten von vorzeitigem Schulaustritt bzw. Schulabbruch sind (Janosz, Archambault, Morizot \& Pagani, 2008). Aus dieser Perspektive illustrieren die vorliegenden Ergebnisse wichtige Bedingungen des Ausschlusses aus dem Bildungssystem, die im vorliegenden Themenheft diskutiert werden.

Hypothesenkonform hängen hohe Elternbelastungen mit dem Leistungsdruck von Eltern gegenüber ihren Kindern positiv, mit der autonomieorien- 
tierten Hilfe jedoch negativ zusammen. Diese Zusammenhänge wurden aber querschnittlich überprüft und sollten nicht kausal interpretiert werden. In Übereinstimmung mit Belsky (1984) unterstützen belastete Eltern ihre Kinder weniger empathisch, indem sie sie weniger zur autonomen Problemlösung von schulischen Aufgaben ermutigen und sich weniger mit dem Lernprozess ihrer Kinder auseinandersetzen. Leistungsdruck korrespondiert positiv mit der Veränderung der Leistungs- und Lernzielorientierung. Damit führen hohe Belastungen der Eltern nicht zu einer Abnahme der Leistungs- und Lernzielorientierung und wirken sich insofern nicht nachteilig auf die Schülermotivation aus. Allerdings zeigen die Ergebnisse, dass hohe Belastungen von Eltern, vermittelt durch das Erziehungsverhalten, die autonome Auseinandersetzung mit Lernprozessen reduzieren, was die intrinsische Lernmotivation und die Schülerleistungen beeinträchtigt (Cheung \& Pomerantz, 2012).

Neu ist die vergleichende Beschreibung der Wirkung des Elternverhaltens auf die Lern- und Leistungszielorientierung in der Sekundarstufe I und bei Schulübergängen. Obwohl sich die Situation in der Sekundarstufe I und bei Schulübertritten in vielerlei Hinsicht unterscheidet, ist das Zusammenhangsmuster von Elternbelastungen auf Erziehungsstil und Lern- und Leistungszielorientierung dasselbe. Die Universalitätsannahme konnte bestätigt werden. Interessanterweise sind die Elternbelastungen während der Schultransition ihrer Kinder nicht höher als nach Eintritt in der Sekundarstufe I. In Abweichung zu Bronfenbrenner (1981), der Schultransitionen als herausfordernde Entwicklungsaufgabe und Belastung konzipiert hat, finden sich in der vorliegenden Studie keine Folgen der Schultransition auf das Belastungserleben.

In Übereinstimmung mit früheren Studien ist die Stabilität der Lern- und Leistungszielorientierung von Kindern über die Zeit hoch (Pintrich et al., 2003). Interessanterweise unterscheidet sich diese Stabilität nicht zwischen der Gruppe der Schülerinnen und Schüler in einer Transitionssituation und der Gruppe, die bereits in der Sekundarstufe I ist. Dies zeigt sich sowohl im Hinblick auf die individuelle Position in der Vergleichsgruppe (Stabilitätskorrelation) als auch in der Abnahme der durchschnittlichen Lern- und Leistungszielorientierung in beiden Gruppen. Der Abfall der Lernzielorientierung beginnt bei der Transition in die Sekundarstufe I und setzt sich in der Sekundarstufe I fort (vgl. auch Frenzel, Pekrun, Dicke \& Goetz, 2012). Die Ergebnisse belegen, dass Eltern durch autonomieorientierte Hilfe und leistungsorientierten Druck diesen sinkenden Trend begünstigen oder aber bremsen können.

Midgley et al. (2001) schlugen eine Differenzierung der Leistungszielorientierung in Vermeidung und Annäherung vor. Diese Differenzierung wurde wegen konzeptueller Unschärfe kritisiert (Conley, 2012), weshalb sie in der vorliegenden Untersuchung nicht aufgegriffen wurde. Damit verbunden ist die Einschränkung, dass nur ein globales Mass für Leistungsorientierung berücksichtigt worden ist. Zudem ist wünschenswert, dass die präsentierten Befunde für den Übergang in die Sekundarstufe II überprüft werden. Studien belegten 
bereits die hohe Bedeutung der Leistungsmotivation für die Planung der Ausbildungswahl und den nicht linearen Verlauf der Lern- und Leistungsmotivation beim Übergang in die Sekundarstufe II (z.B. Neuenschwander et al., 2012). Es sind aber keine Studien zum Einfluss des elterlichen Erziehungsverhaltens auf die Entwicklung der Lern- und Leistungszielorientierung beim Übergang in die berufliche Grundbildung bekannt. Dazu wäre zukünftige Forschung wünschenswert. Immerhin zeigen die vorliegenden Ergebnisse, wie Belastungen von Eltern, vermittelt über ihren Erziehungsstil, die Lernziel- und Leistungszielorientierung in der Sekundarstufe I und beim Eintritt in die Sekundarstufe I beeinflussen.

\section{Anmerkung}

1 Dank geht an den Schweizerischen Nationalfonds für den finanziellen Beitrag an diese Studie (Projektnummer 100013_134594/1). Ich danke herzlich Dr. Benno Rottermann, Stephan Rösselet, Edith Niederbacher für die Mitarbeit bei der Projektdurchführung.

\section{Literatur}

Belsky, J. (1984). The determination of parenting: A process model. Child Developemt. 55, 83-96.

Bronfenbrenner, U. (1981. Die Oekologie der menschlichen Entwicklung. Stuttgart: KlettCotta.

Castro, M., Expósito-Casas, E., López-Martín, E., Lizasoain, L., Navarro-Asencio, E. \& Gaviria, J. L. (2015). Parental involvement on student academic achievement: A meta-analysis. Educational Research Review, 14, 33-46. doi:10.1016/j.edurev.2015.01.002

Cheung, C. S.-S. \& Pomerantz, E. M. (2012). Why does parents' involvement enhance children's achievement? The role of parent-oriented motivation. Journal of Educational Psychology, 104, (3), 820-832. doi:10.1037/a0027183

Cohen, S., Kamarck, T. \& Mermelstein, R. (1983). A global measure of perceived stress. Journal of Health and Social Behavior, 24, (4), 385-396. doi:10.2307/2136404

Conley, A. M. (2012). Patterns of motivation beliefs: Combining achievement goal and expectancy-value perspectives. Journal of Educational Psychology, 104, (1), 32-47. doi:10.1037/ a0026042

Duchesne, S. \& Ratelle, C. (2010). Parental behaviors and adolescents' achievement goals at the beginning of middle school: Emotional problems as potential mediators. Journal of Educational Psychology, 102, (2), 497. doi:10.1037/a0019320

Frenzel, A. C., Pekrun, R., Dicke, A.-L. \& Goetz, T. (2012). Beyond quantitative decline: Conceptual shifts in adolescents' development of interest in mathematics. Developmental Psychology, 48, (4), 1069-1082. doi:10.1037/a0026895

Gonzalez-DeHass, A. R., Willems, P. P. \& Holbein, M. F. D. (2005). Examining the relationship between parental involvement and student motivation. Educational Psychology Review, 17, (2), 99-123. doi:10.1007/s10648-005-3949-7

Graham, J. W. (2009). Missing data analysis: Making it work in the real world. Annual Review of Psychology, 60, 549-576. doi:0.1146/annurev.psych.58.110405.085530

Grolnick, W. S. (2009). The role of parents in facilitating autonomous self-regulation for education. Theory and Research in Education, 7, (2), 164-173. doi:10.1177/1477878509104321

Grolnick, W. S., Friendly, R. W. \& Bellas, V. M. (2009). Parenting and children's motivation at school. In K. Wentzel, A. Wigfield \& D. Miele (Eds.), Handbook of Motivation at School (pp. 279-300). New York: Routledge. 
Gurland, S. T. \& Grolnick, W. S. (2005). Perceived threat, controlling parenting, and children's achievement orientations. Motivation and Emotion, 29, (2), 103-121. doi:10.1007/ s11031-005-7956-2

Janosz, M., Archambault, I., Morizot, J. \& Pagani, L. S. (2008). School engagement trajectories and their differential predictive relations to dropout. Journal of Social Issues, 64, (1), 21-40.

Little, T. D. (2013). Longitudinal structural equation modeling. New York: Guilford Press.

Midgley, C., Kaplan, A. \& Middleton, M. (2001). Performance-approach goals: Good for what, for whom, under what circumstances, and at what cost? Journal of Educational Psychology, 93, (1), 77-86. doi:10.1037/0022-0663.93.1.77

Midgley, C., Maehr, M., Hruda, L., Anderman, E., Anderman, L. \& Freeman, K. (2000). Manual for the Patterns of Adaptive Learning Scales. Retrieved from Ann Arbor: http:// www.umich.edu/ - pals/PALS\%202000_V13Word97.pdf. 15. Januar 2017.

Muthén, L.K. \& Muthén, B. O. (1998-2012). MPlus user's guide. Los Angeles: Muthén \& Muthén.

Neuenschwander, M. P. \& Frank, N. (2011). Entwicklung von Lebenszielen in der Familie. Zeitschrift für Entwicklungspsychologie und Pädagogische Psychologie, 43, (2), 68-77. doi:10.1026/0049-8637/a000036

Neuenschwander, M. P. (im Druck). Anpassungsprozesse beim Übergang in die Sekundarstufe I. In M. P. Neuenschwander \& C. Nägele (Hrsg.), Bildungsverläufe von der Einschulung in die Erwerbstätigkeit. Wiesbaden: VS Verlag für Sozialwissenschaften.

Neuenschwander, M. P., Balmer, T., Gasser, A., Goltz, S., Hirt, U., Ryser, H. \& Wartenweiler, H. (2005). Schule und Familie. Bern: Haupt.

Neuenschwander, M. P., Gerber, M., Frank, N. \& Rottermann, B. (2012). Schule und Beruf. Wiesbaden: VS-Verlag.

Newland, R. P., Crnic, K. A., Cox, M. J., Mills-Koonce, W. R. \& Family Life Project Key, I. (2013). The family model stress and maternal psychological symptoms: mediated pathways from economic hardship to parenting. Journal of Family Psychology, 27, (1), 96-105. doi: $10.1037 / \mathrm{a} 0031112$

Pintrich, P. R., Conley, A. M. \& Kempler, T. M. (2003). Current issues in achievement goal theory and research. International Journal of Educational Research, 39, 319-337. doi:10.1016/j.ijer.2004.06.002

Régner, I., Loose, F. \& Dumas, F. (2009). Students' perceptions of parental and teacher academic involvement: Consequences on achievement goals. European Journal of Psychology of Education, 24, (2), 263-277. doi:10.1007/bf03173016

Roberts, W. L. (1989). Parents' stressful life events and social networks: Relations with parenting and children's competence. Canadian Journal of Behavior Science, 21, 132-146. doi: $10.1037 / \mathrm{h} 0079811$

Sirsch, U. (2000). Probleme beim Schulwechsel. Münster: Waxmann.

Van de Schoot, R., Lugtig, P. \& Hox, J. (2012). A checklist for testing measurement invariance. European Journal of Developmental Psychology, 9, (4), 1-7. doi:10.1080/17405 629.2012 .686740

Wild, E. (1999). Elterliche Erziehung und schulische Lernmotivation. Universität Mannheim.

Wild, E., Remy, K., Gerber, J. \& Exeler, J. (2001). Dokumentation der Skalen und Itemauswahl für den Elternfragebogen zur Lernmotivation und zum emotionalen Erleben ibres Kindes. Bielefeld: Universität Bielefeld.

Schlagworte: Motivation, Elternbelastungen, Erziehungsstil, Transition, Strukturgleichungsmodell, Längsschnitt 


\section{La focalisation sur les objectifs d'apprentissages et de performances lors du passage au secondaire I: résultats d'une étude longitudinale à propos des comportements éducatifs des parents}

\section{Résumé}

Le stress des parents influence les stratégies mises en ouvre par ces derniers pour soutenir leurs enfants dans leurs apprentissages. Ces hypothèses ont été examinées à partir des données issues de l'étude longitudinale "Wirkungen der Selektion». Les sujets du groupe expérimental, constitué de 1096 parents et de leurs enfants fréquentant les $6^{\mathrm{e}}$ et $7^{\mathrm{e}}$ années de l'enseignement secondaire, ainsi que les sujets du groupe contrôle incluant 778 parents et leurs enfants, ont répondu à un questionnaire. Des modélisations en équations structurelles et des analyses multi-groupes ont montré que les parents stressés avaient tendance à exercer davantage de pression pour améliorer les performances scolaires de leur enfant, conduisant ainsi les éducateurs à chercher à accroitre les performances des élèves. Les résultats de recherche montrent encore que les parents seraient tout à fait en mesure d'exhorter leur enfant à s'engager dans leurs apprentissages et à mobiliser leurs efforts et attention pour $\mathrm{y}$ arriver.

Mots-clés: Motivation, stress parental, éducation des parents, transition, modélisation en équations structurelles, étude longitudinale

\section{Obiettivi di padronanza e di prestazione durante la transizione al secondario I: Risultati longitudinali sull'influenza del gravame percepito dai genitori e il loro comportamento}

\section{Riassunto}

Il gravame percepito dai genitori influenza le loro strategie di sostegno e l'orientamento agli obiettivi di padronanza e di prestazione dei figli. Queste ipotesi sono state verificate in un'analisi longitudinale dei dati raccolti nello studio: «Effetti della selezione» (WiSel - Wirkungen der Selektion). Sono stati intervistati 1096 genitori e i loro figli nel VI e VII anno scolastico del secondario I e come gruppo di controllo 778 genitori e i loro figli residenti in cantoni in cui la transizione dal primario al secondario I avviene dopo il VI anno scolastico. Confrontando i gruppi con l'ausilio di modelli di equazioni strutturali, si può dimostrare che i genitori oberati in ambedue i gruppi esercitano sui figli una pressione di tipo prestazionale. Questo tipo di pressione è un predittore dell'orientamento agli obiettivi dei figli. I risultati forniscono informazioni su come i genitori possono contrastare la diminuzione dell'orientamento agli obiettivi di padronanza e di prestazione. 
Parole chiavi: Motivazione, genitori oberati, stile educativo, transizione, modelli di equazioni strutturali, analisi longitudinali.

\section{Mastery Goal and Performance Goal Orientation in the Transition to Lower Secondary Education: Longitudinal Findings on the Importance of Parental Stress and Educational Behavior}

\section{Summary}

Parental stress influences the support strategies they implement to support their children's learning. These hypotheses were examined using data from the longitudinal study "Wirkungen der Selektion". The subjects in the experimental group, consisting of 1096 parents and their children in lower secondary education grades 6 and 7, as well as the control group including 778 parents and their children, answered a questionnaire. Structural equation modelling and multi-group analyses showed that in both groups stressed parents showed higher performance oriented pressure, leading educators to seek to increase the student's performance. The results indicate how parents can counteract decreasing mastery and performance goal orientation.

Keywords: Motivation, parental stress, parenting, transition, structural equation model, longitudinal study 